\title{
Fixation of Posterior Malleolus is Enough for Syndesmotic Stability: Fact or Fiction?
}

\author{
Emre Baca ${ }^{1}$, (D) Nezih Ziroğlu² \\ 1 University of Health Sciences Turkey, Bakırköy Dr. Sadi Konuk Training and Research Hospital, Clinic of Orthopaedics and Travmatology, Istanbul, Turkey \\ ${ }^{2}$ Beylikduzu State Hospital, Clinic of Orthopaedics and Travmatology, istanbul, Turkey
}

\section{Abstract}

Objective: This study aimed to evaluate syndesmotic stability following anatomic reduction and fixation of the posterior malleolus (PM) of ankle fractures with syndesmotic instability, without utilizing a classical syndesmotic screw.

Methods: We have retrospectively evaluated patients with PM fracture and syndesmotic displacement between September 2012 and May 2017. The inclusion criteria were as follows: patients with (1) PM fracture, either isolated or a part of bi-/trimalleolar fracture with syndesmotic instability; (2) fractures fixed through a posterior approach; (3) fractures fixed with either screw or plate-screw combination; and (4) Bartonicek type 2-5 fractures. Among 145 patients, 41 (27 female, 14 male) met the inclusion criteria. The average age was 42.65 years, and the mean follow-up time was 19.41 months. Ankle fractures were classified according to the Weber classification, while PM fractures according to the Bartonicek classification. Perioperative reduction was evaluated by anteroposterior, lateral, and mortise views. Perioperative and postoperative stability was evaluated using Cotton and fibular translation tests. Postoperative syndemotic reduction was evaluated with computed tomography (CT) scan according to Dikos and Futamura.

Results: According to the Weber classification, 22 were type B, 17 type C, and 2 unclassified because they did not get lateral malleolus fracture. According to Bartonicek classification, 17 fractures (41.5\%) were type 2, 14 (34.1\%) type 3, 9 (22\%) type 4, and 1 (2.4\%) type 5. All patients had unilateral fractures. On postoperative CT scan evaluation, 38 (92.68\%) patients got syndesmotic reduction, and 3 (7.32\%) got syndesmotic malreduction.

Conclusion: This study demonstrated that if appropriate surgical principles are followed, and meticulous attention paid for reduction and fixation, fixing only the PM achieves syndesmotic stability for patients with PM fracture and syndesmotic diastasis. The indication for PM fixation should not be based on size alone. Not all, but PM fractures with syndesmotic displacement should be operated.

Keywords: Posterior malleolus, syndesmosis, fixation

\section{INTRODUCTION}

Ankle fractures are common injuries (1). Posterior malleolus (PM) fractures are present in $10 \%$ to $44 \%$ of all ankle fractures (2). The presence of a posterior fragment is well-established to have a negative effect on the clinical outcome of ankle fractures (3).

Classically, for posterior fragments $>25 \%-33 \%$ of the anteroposterior diameter of the articular surface of the distal tibia as measured on a plain lateral radiograph, open reduction and internal fixation of the fragment should be performed $(4,5)$.
However, plain radiography poorly assesses the trimalleolar ankle fractures (6). But in the last decade, the indication for surgery of PM has been changed. Syndesmotic stability, the involvement of the fibular notch, and the presence of intercalary fragments are more important than the size of the fracture and the amount of the fractured articular surface (7).

The syndesmosis represents a complex ligamentous structure formed by four ligaments: anterior inferior tibiofibular ligament (AITFL), interosseous ligament (IOL), posterior inferior 
tibiofibular ligament (PITFL), and inferior transverse ligament (8). Among them, PITFL forms the main resistance against diastasis with $42 \%$, followed by AITFL with $35 \%$ and IOL with $22 \%$ (9). Biomechanical studies suggest the restoration of the posterior aspect of the tibiofibular ligament with the fixation of the PM, obviating the need for syndesmotic stabilization $(9,10)$. A cadaveric study showed that PITFL remained attached to the PM fragment in cases of PM fracture (10).

A preoperative computed tomography (CT) scan is imperative to evaluate the fragment size, comminution, articular impaction, and syndesmotic disruption (11). With the frequent use of the CT scan to assess the 3D geometry of PM fractures, an increasing number of authors recommend internal fixation of any displaced PM involving the fibular notch regardless of its size because it recreates the notch for fibular reduction and substantially contributes to syndesmotic stability (7).

This study aimed to evaluate syndesmotic integrity following anatomic reduction and fixation of the PM of ankle fractures with syndesmotic instability, without utilizing a classical syndesmotic screw. Our hypothesis was that fixing only the PM would result in distal tibiofibular reduction on postoperative CT scans and stability on clinical tests.

\section{METHODS}

We have retrospectively evaluated patients with PM fracture and syndesmotic instability between September 2012 and May 2017. The inclusion criteria were as follows: patients with (1) PM fracture, either isolated or a part of bi-/trimalleolar fracture with syndesmotic instability; (2) fractures fixed through a posterior approach; (3) fractures fixed with either screw or platescrew combination; and (4) Bartonicek type 2-5 fractures. The exclusion criteria, on the other hand, were as follows: patients (1) with pathologic fractures, (2) with posterior pilon fractures (3) with less than 1-year follow-up, (4) without preoperative or postoperative CT scans, (5) with fractures fixed anteriorly, (6) with a laterally placed syndesmotic screw, and (7) with Bartonicek type 1 fractures.

The study was approved by the institutional review board of Bakırköy Training and Research Hospital (2017-18-30).

Informed consent was taken from all patients.

All patients who have been included in the study were evaluated preoperatively and postoperatively using X-rays (anteroposterior, lateral, and mortise views) and CT scans. Preoperative syndesmotic stability was evaluated by mortise views and Cotton and fibular translation tests. Syndesmotic reduction was evaluated according to Dikos et al. (12), with three measurements chosen to contain three deforming vectors (13). Tibiofibular clear space was measured as the interval between medial fibula and the tip of the posterior tibial tubercle, which reflects mediolateral diastasis. Anterior tibiofibular interval is measured as the distance between the anterior tibia and the anterior fibula to reflect anteroposterior deviation of the fibula. Finally, $\theta$ fib is measured for the rotational malalignment of the fibula, which is the angle formed between a tangential line to the anterior and posterior tibial tubercles and a line through the anterior and posterior fibular tubercles. All measurements were done using transverse CT scans $1 \mathrm{~cm}$ proximal from the ankle joint level and bilaterally, to use the noninjured side as a control group for the calculation of the difference between the injured and noninjured sides (Figure 1, Table 1).

Ankle fractures were classified according to the Weber classification. Infrasyndesmotic lateral malleolus fractures were classified as type A, syndesmotic fractures as type B, and supra syndesmotic fractures as type C. But this system is not sufficient, because it does not include PM. Therefore, fractures were additionally classified according to Bartonicek classification: extraincisural fragments were classified as type 1, posterolateral fragments as type 2, posteromedial two-part fragments as type 3 , large posterolateral triangular fragments as type 4 , and irregular osteoporotic fractures as type 5 (7).

All patients underwent operation on the prone position under either general or spinal anesthesia. A thigh tourniquet was used to prevent bleeding. A modified posterolateral approach was used to access PM and, if necessary, lateral malleolus (Figure 2). Standard posterolateral approach gives a nice view of the PM fragment and maintains a window for anatomical reduction and rigid fixation (14). A modification was used to avoid excessive force during ecartation. The sural nerve and lesser saphenous vein were protected during superficial dissection (Figure 3). The PM was reached between the flexor hallucis longus and peroneus longus interval, which were reduced and fixed in the first place. Care was taken to prevent posterior-inferior tibiofibular ligament displacement (Figure 4), followed by, from the same approach, the reduction and lateral fixation of lateral malleolus fracture using the advantage of a modified approach. The medial

\begin{tabular}{|l|l|l|}
\hline \multicolumn{3}{|l|}{ Table 1. Normal side-to-side differences } \\
\hline & Mean variability & Maximum variability \\
\hline TFCS $(\mathrm{mm})$ & $0.7 \pm 0.6(0.0-2.7)$ & 1.9 \\
\hline ATF $(\mathrm{mm})$ & $0.8 \pm 0.7(0.0-3.0)$ & 2.3 \\
\hline$\theta$ fib $\left(^{\circ}\right)$ & $2.9 \pm 1.8(0.2-6.4)$ & 6.5 \\
\hline TFCS: Tibiofibular clear space, ATF: Anterior tibiofibular interval \\
\hline
\end{tabular}




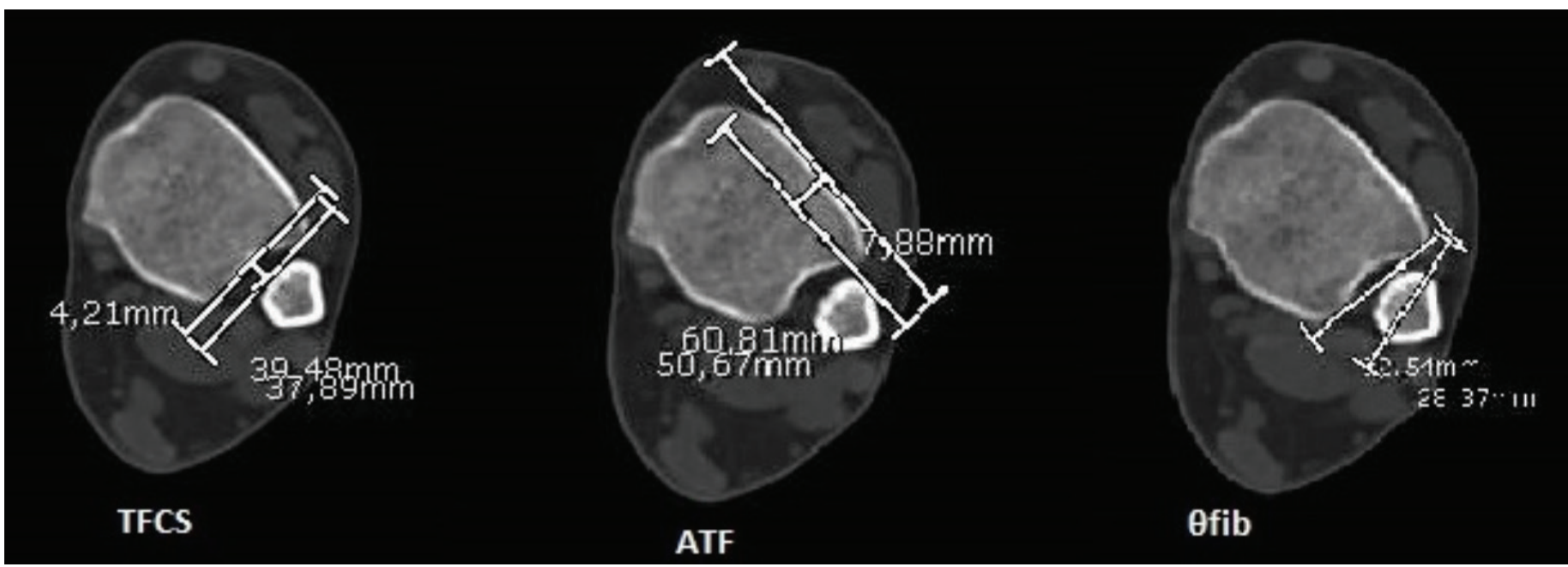

Figure 1. Measurements according to Dikos

TFCS: Tibiofibular clear space, ATF: Anterior tibiofibular interval

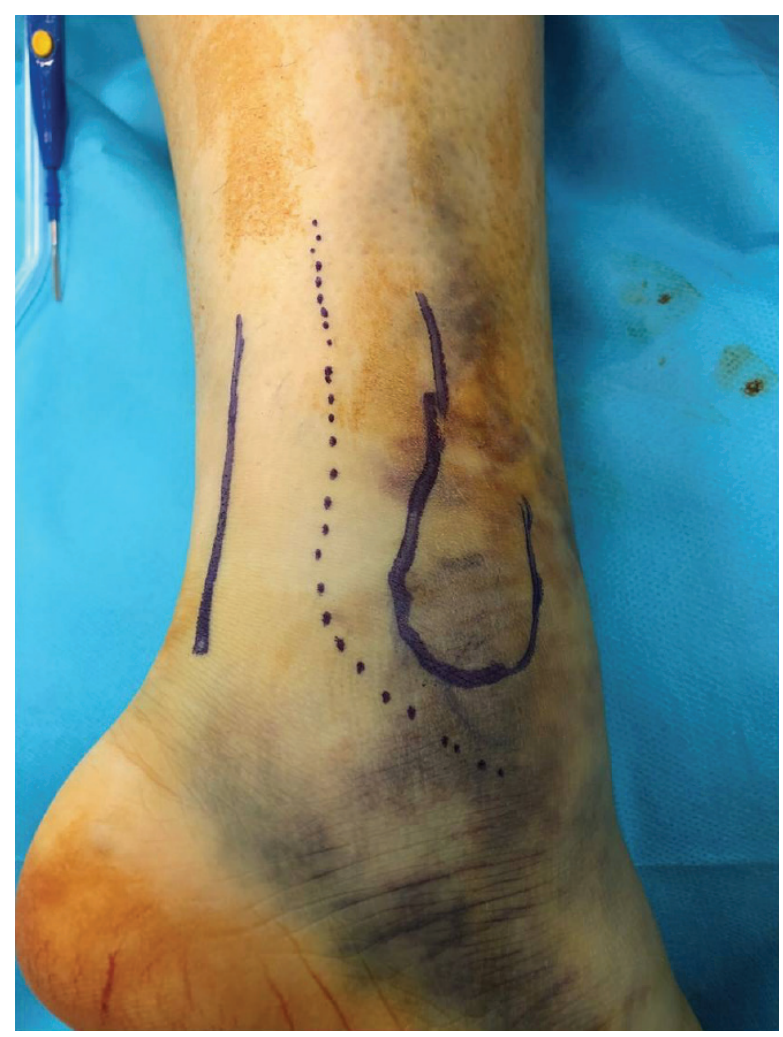

Figure 2. Modified posterolateral approach. The straight line shows the lateral border of the Achilles tendon. The lateral fibula is marked. Between these lines, the dotted line with a curve on the tip of the lateral malleolus shows a modified approach

malleolus fracture treatment used a separate medial curve incision. All reductions and fixations were controlled using the intraoperative C-arm; the anteroposterior, lateral, and mortise views were obtained for this purpose. Intraoperatively, after the fixation of all fractures, the fibular translation and Cotton tests evaluated the syndesmotic integrity. With the Cotton test,

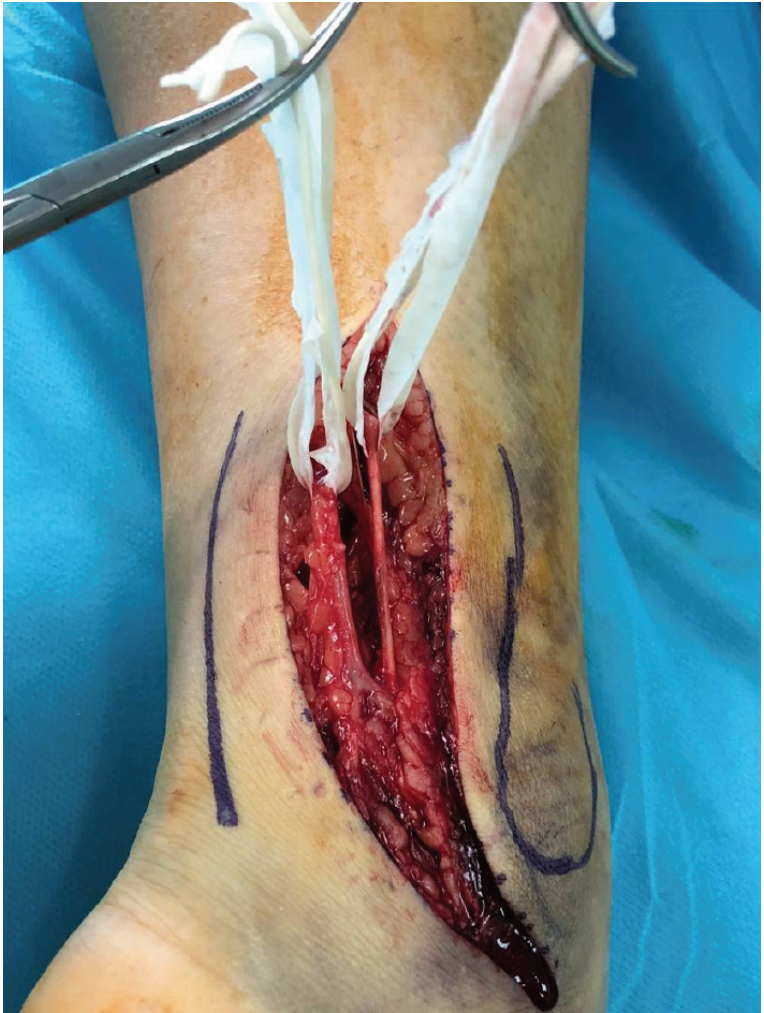

Figure 3. Sural nerve and lesser saphenous nerve

medial and lateral forces are applied to the talus with the ankle in the neutral position. The fibula translation test examines the fibula in the anteroposterior direction, which is positive when an excessive amount of translation is felt compared to the opposite ankle (15). 
Postoperative X-rays and CT scans were taken to evaluate the quality of fracture and syndesmosis reduction that was measured according to Dikos et al. (12) and Futamura et al. (13).

Postoperatively, all fractures were stabilized for 6 weeks with a short leg splint. Regular clinic assessments were made at 2 weeks, 6 weeks, 10 weeks, 14 weeks, 6 months, and 12 months, with radiologic assessments on every clinical control except for the second week. CT scans were taken in the early postoperative period and in the final follow-up visit. After 6 weeks, progressive weight-bearing was started according to the healing status shown in clinic X-ray follow-ups.

Cotton and fibular translation tests evaluated the postoperative syndesmotic stability.

Each patient and their related radiological imaging were evaluated individually, with their result noted as a percentage value. No other statistical measurement was used.

\section{RESULTS}

Among 145 patients, 41 met the inclusion criteria. Among the excluded 101 patients, 85 were conservatively treated, 1 got a pathologic fracture, 2 were fixed anteriorly, 6 got an additional syndesmotic screw, and 10 had a $<1$-year follow-up period.

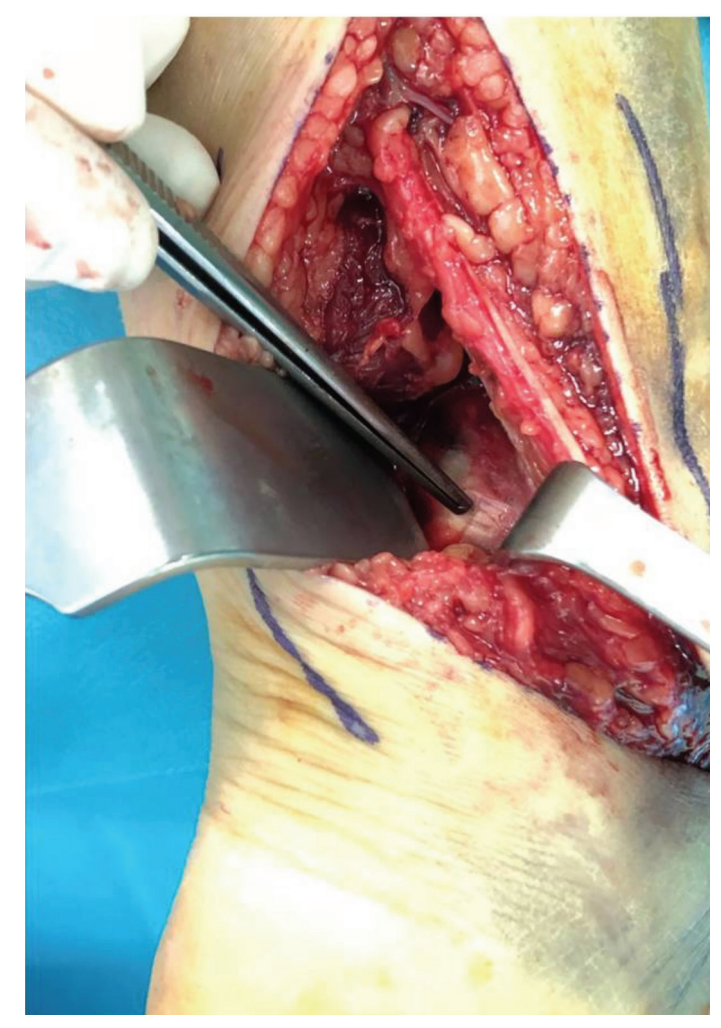

Figure 4. PITFL on the tip of forceps

PITFL: Posterior inferior tibiofibular ligament
Also, 27 were female and 14 male, with an average age of 42.65 (range, 15-75) years. All patients had unilateral fractures, with 24 on the right side and 17 on the left side. The mean follow-up time was 19.41 (range, 12-67) months.

According to the Weber classification, 22 were type B, 17 type $C$, and 2 unclassified because they did not get lateral malleolus fracture.

According to Bartonicek classification, 17 fractures (41.5\%) were type 2, 14 (34.1\%) type 3, 9 (22\%) type 4, and 1 (2.4\%) type 5.

On postoperative CT scan evaluation, 38 (92.68\%) patients got and maintained the syndesmotic reduction upon the final follow-up visit. Three (7.32\%) patients got syndesmotic malreduction. After patient evaluation, one got malreduced syndesmosis because of the malreduced PM (Figure 5). One patient got ankle arthrosis because of complicated aberrant heterotopic ossification, causing syndesmotic diastasis, which can be interpreted as a complication rather than an early malreduction (Figure 6). The last one had a fixation problem, and the screw used to fix PM penetrated the tibiofibular space, avoiding syndesmotic reduction (Figure 7). According to Bartonicek classification, these three were distributed one by one to types 2-4.

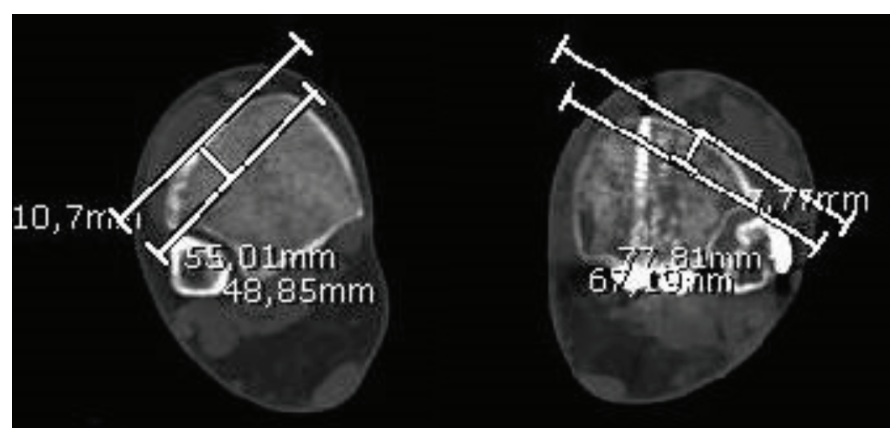

Figure 5. Malreduced syndesmosis secondary to malreduced PM PM: Posterior malleolus

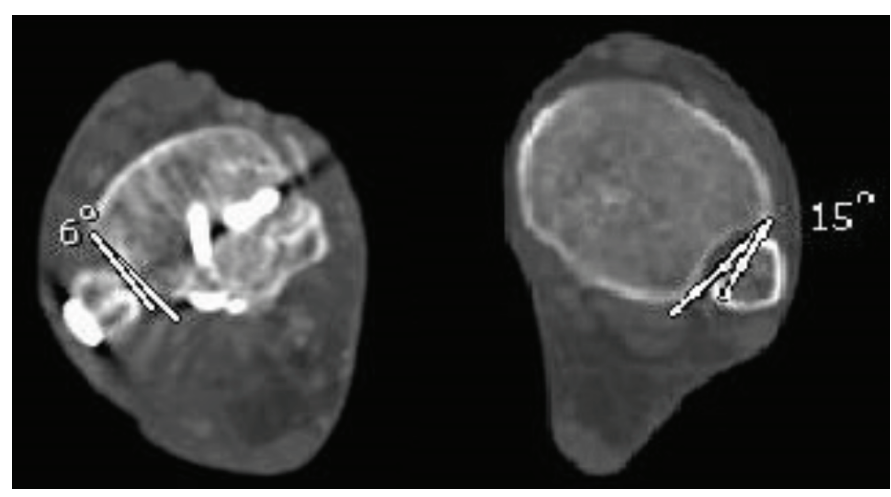

Figure 6. Malreduced syndesmosis secondary to arthrosis 
The three malreduced patients were unstable when clinically evaluated by the Cotton and fibular translation tests postoperatively.

No major intraoperative complications were noted. Three patients developed a superficial infection, which was managed using local dressing and antibiotics. Nonunions or implant failure was not seen as well.

\section{DISCUSSION}

This study demonstrated that if appropriate surgical principles are followed, and meticulous attention paid for reduction and fixation, fixing only the PM achieves syndesmotic reduction, as well as stability for patients with PM fracture and syndesmotic diastasis, and diminishes the need for an extra syndesmotic screw. Our surgical principles for PM fractures with syndesmotic displacement are as follows: prone positioning, distally curved modified posterolateral incision to avoid excessive force during ecartation, starting fixation from PM and then moving to lateral and medial malleoli if exists, perioperative Cotton and fibular translation tests for stability, and AP, lateral, and mortise views for reduction evaluation. In this study, syndesmotic reduction was achieved $92.68 \%$ (38/41) of the time, and two of the three malreduced syndesmoses could be avoided.

In the past, the indication for PM surgery was a fragment size $>25 \%$ to $33 \%$ of the articular surface and displacement $>2 \mathrm{~mm}$ $(4,5)$. But over the last decades, these indications have evolved. Studies suggest that the involvement of the fibular notch, impacted intercalary articular fragments, and syndesmotic instability has greater therapeutic relevance than the size of the fragment and amount of the fractured articular surface (7). Moreover, plain $\mathrm{X}$-rays have been shown to be insufficient in evaluating the nature of the fracture and syndesmotic stability (6). Therefore in this study, we used pre- and postoperative CT scans in evaluating the fracture pattern and syndesmotic stability. For indication, we take care of the recent evolutions.

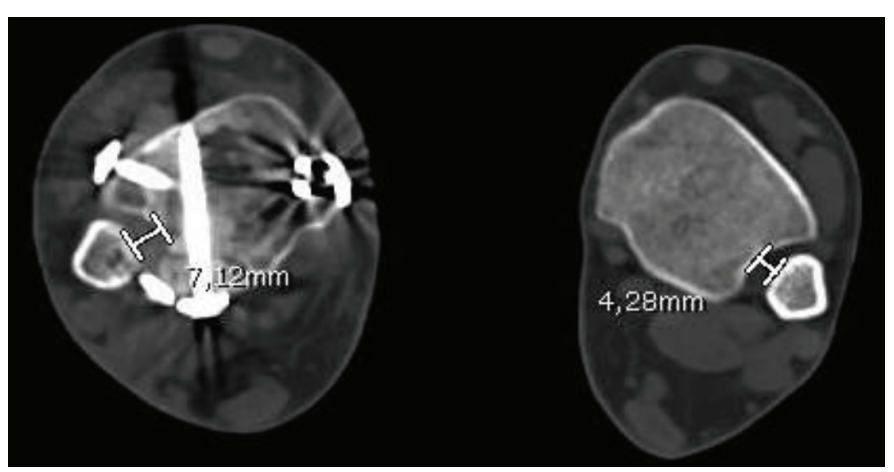

Figure 7. Malreduced syndesmosis secondary to malfixation
The size was not taken care because even the mere presence of a small posterior fragment causes a negative effect on ankle fracture outcomes (3). In this study, all patients with fibular notch, syndesmotic instability, and impacted intercalary articular fragment involvement underwent operation.

According to the Weber classification, 22 were type B, 17 type $C$, and 2 unclassified because the first one was an isolated PM fracture with syndesmotic displacement and the second were medial and PM fractures with syndesmotic displacement. The number of type B fractures may be confusing, but Tornetta has shown an incidence of $39 \%$ syndesmotic instability with type B fractures (16).

According to Bartonicek classification, 17 fractures (41.5\%) were type 2, 14 (34.1\%) type 3, 9 (22\%) type 4, and 1 (2.4\%) type 5, which are different from the study of Bartonicek et al. (7), possibly because of the patient group we evaluated. In this study, only surgically treated patients were included. Therefore, there are no Bartonicek type 1 fractures.

All the operations were made in the prone position, and fixation started from the PM. In their comparative series, Miller et al. (17) compared supine and prone positioning for the operation, concluding that $24.5 \%$ of the patients in the supine position needed an extra posterior fixation and the rate of syndesmotic instability was reduced when prone positioning and direct fixation of the PM were first performed. Also, fixing the PM in the first step reduces the distal fibula to the exact length through the PITFL (1).

Indirect reduction using ligamentotaxis and fixation from anterior to posterior can be used for PM fractures. But direct reduction and fixation from the posterior side have been shown to provide better reduction quality and functional outcomes $(18,19)$. Also, it is more stable than the anteroposterior fixation $(14,15)$.

Perioperative and postoperative syndesmotic stability control was achieved by using fibular translation and Cotton tests, which were chosen for this study because they were two of the three tests advised by the ESSKA-AFAS consensus panel (20). At the time of surgery, all patients showed syndesmotic stability. But on the postoperative CT scan evaluation, 3 patients got syndesmotic malreduction, and 38 achieved stability. This rate of stability is consistent with the literature (17). Of the three malreduced patients, two could be avoided. One of them got a screw in the tibiofibular space, causing syndesmotic diastasis. The other one was a PM malreduction, causing syndesmotic malreduction. They were cases operated at the beginning of the learning curve. 
Perioperative X-ray examination must be much more precise to prevent these complications, and the tests should be done bilaterally.

Three (7.32\%) patients got syndesmotic malreduction. According to Bartonicek classification, these three were distributed one by one to types 2-4. The number of malreduction is the same for all the types. But when looking at the percentages of malreduction, type 4 has 11\%; type 3, 7\%; and type 2, 5.8\%. The incidence rises with the type, probably because the energy amount rises with the type of the fracture (7).

In their study, Miller et al. (17) evaluated the effect of PM fixation on syndesmotic integrity. But the current study differs from Miller et al. (17) in important ways. First and foremost, they have decided prone or supine positioning preoperatively according to the percentage of PM and used $25 \%$ as a fixation indication for PM. They also did not use a preoperative CT scan for surgical decision-making. Secondly, in Miller et al. (17) study, they did not give the sequence for the surgery. We operated patients following the posterior, lateral, and medial malleolus sequences.

According to Gardner et al. (10), the fixation of PM alone gives $70 \%$ syndesmotic stability. In our study, all appropriately fixed PM gave enough syndesmotic stability according to perioperative stress testing and postoperative CT scans.

Another advantage of fixing only PM for syndesmotic integrity is negating any additional screw or tightrope for the stabilization of syndesmosis. Also, it diminishes the need for screw removal, and postoperative rehabilitation can be quicker (1).

\section{Study Limitations}

Our study has some weaknesses and limitations: the retrospective design of the study, no follow-up with questionnaires, and small sample size.

\section{CONCLUSION}

This study shows that for the fixation of PM, instead of articular involvement, the size of the fragment and displacement, the involvement of the fibular notch, impacted intercalary articular fragments, and syndesmotic instability should be used. We suggest that not all PM fractures but those with syndesmotic displacement should be approached surgically. Second, the fixation of PM without an additional syndesmotic screw is stable enough.

\section{Ethics}

Ethics Committee Approval: The study was approved by the institutional review board of Bakırköy Training and Research Hospital (2017-18-30).
Informed Consent: Informed consent was taken from all patients.

Peer-review: Externally and internally peer-reviewed.

\section{Authorship Contributions}

Surgical and Medical Practices: E.B., N.Z., Concept: E.B., Design: E.B., Data Collection or Processing: N.Z., Analysis or Interpretation: N.Z., Literature Search: E.B., N.Z., Writing: E.B., N.Z.

Conflict of Interest: No conflict of interest was declared by the authors.

Financial Disclosure: The authors declared that this study received no financial support.

\section{REFERENCES}

1. Solan MC, Sakellariou A. Posterior malleolus fractures: worth fixing. Bone Joint J 2017;99:1413-9.

2. Wang Xu, Yin J, Zhang C, Wang C, Geng X, Ma X, et al. Biomechanical study of screw fixation and plate fixation of a posterior malleolar fracture in a simulation of the normal gait cycle. Foot Ankle Int 2017;38:1132-8.

3. Tejwani NC, Pahk B, Egol KA. Effect of posterior malleolus fracture on outcome after unstable ankle fracture. J Trauma 2010;69:666-9.

4. Van den Bekerom MP, Haverkamp D, Kloen P. Biomechanical and clinical evaluation of posterior malleolar fractures. A systematic review of the literature. J Trauma 2009;66:279-84.

5. McLaughlin H. Injuries of the ankle. Trauma; 1959 p. 357-60.

6. Meijer DT, Doornberg JN, Sierevelt IN, Mallee WH, van Dijk CN, Kerkhoffs GM, et al. Guesstimation of posterior malleolar fractures on lateral plain radiographs. Injury 2015;46:2024-9.

7. Bartonicek J, Rammelt S, Tucek M. Posterior malleolar fractures: changing concepts and recent developments. Foot Ankle Clin 2017:22:125-45.

8. Lilyquist M, Shaw A, Latz K, Bogener J, Wentz B. Cadaveric analysis of the distal tibiofibular syndesmosis. Foot Ankle Int 2016;37:882-90.

9. Ogilvie-Harris DJ, Reed SD, Hedman TP. Disruption of the ankle syndesmosis: biomechanical study of the ligamentous restraints. Arthroscopy 1994;10:558-60.

10. Gardner MJ, Brodsky A, Briggs SM, Nielson JH, Lorich DG. Fixation of posterior malleolar fractures provides greater syndesmotic stability. Clin Orthop Relat Res 2006;447:165-71.

11. Tenenbaum S, Shazar N, Bruck N, Bariteau J. Posterior malleolus ractures. Orthopedic Clin North Am 2017:48:81-9.

12. Dikos GD, Heisler J, Choplin RH, Weber TG. Normal tibiofibular relationships at the syndesmosis on axial CT imaging. J Orthop Trauma 2012;26:433-8.

13. Futamura K, Baba T, Mogami A, Morohashi I, Kanda A, Obayashi O, et al. Malreduction of syndesmosis injury associated with malleolar ankle fracture can be avoided using Weber's three indexes in the mortise view. Injury 2017;48:954-9. 
14. Verhage SM, Boot F, Schipper IB, Hoogendoorn JM. Open reduction and internal fixation of posterior malleolar fractures using the posterolateral approach. Bone Joint J 2016;98:812-7.

15. Mulligan EP. Evaluation and management of ankle syndesmosis injuries. Phys Ther Sport 2017;12:57-69.

16. Stark E, Tornetta P, Creevy WR. Syndesmotic instability in Weber B ankle fractures: a clinical evaluation. J Orthop Trauma 2007;21:643-6.

17. Miller MA, McDonald TC, Graves ML, Spitler CA, Russell GV, Jones LC, et al. Stability of the syndesmosis after posterior malleolar fracture fixation. Foot Ankle Int 2018;39:99-104.
18. Shi H, Xiong, J, Chen YX, Wang JF, Qiu, XS, Huang J, et al. Comparison of the direct and indirect reduction techniques during the surgical management of posterior malleolar fractures. BMC Musculoskeletal Disorders 2017;18:109.

19. Anwar A, Zhang Z, Lv D, Lv G, Zhao Z, Wang Y, et al. Biomechanical efficacy of AP, PA lag screws and posterior plating for fixation of posterior malleolar fractures: a three dimensional finite element study. BMC musculoskeletal disorders 2018:19:73.

20. Van Dijk CN, Longo UG, Loppini M, Florio P, Maltese L, Ciuffreda M, et al. Classification and diagnosis of acute isolated syndesmotic injuries: ESSKA-AFAS consensus and guidelines. Knee Surg Sports Traumatol Arthrosc 2016;24:1200-16. 